\title{
REAÇÕES DE CARBOCICLIZAÇÃO RADICALAR DE ORTO-IODOALILOXIBENZOATOS DERIVADOS DE D-GLICOSE E D-GALACTOSE E COMPARAÇÃO COM AS REAÇÕES DE SEUS ANÁLOGOS BENZAMIDAS
}

Ildefonso Binatti, Rosemeire Brondi Alves e José Dias de Souza Filho

Departamento de Química, Instituto de Ciências Exatas, Universidade Federal de Minas Gerais, CP 702, 31270-901 Belo Horizonte - MG

Danielle Ferreira Dias, Maria Auxiliadôra Fontes Prado* e Ricardo José Alves

Departamento de Produtos Farmacêuticos, Faculdade de Farmácia, Universidade Federal de Minas Gerais, Av. Olegário Maciel, 2630, 30180-112 Belo Horizonte - MG

Recebido em 28/10/04; aceito em 21/3/05; publicado na web em 24/8/05

\begin{abstract}
RADICAL CARBOCYCLIZATION REACTIONS OF ORTHO-IODOALLYLOXYBENZOATE DERIVATIVES OF D-GLUCOSE AND D-GALACTOSE AND COMPARISON WITH THE REACTIONS OF THEIR BENZAMIDE ANALOGS. Two orthoiodoallyloxybenzoates, methyl 4-O-allyl-2,3-di- $O$-benzyl-6- $O$-(2-iodobenzoyl)- $\alpha$-D-glucopyranoside (3) and methyl 4- $O$-allyl-2,3di- $O$-benzyl-6- $O$-(2-iodobenzoyl)- $\alpha$-D-galactopyranoside (4) were synthesized in seven conventional steps from methyl $\alpha$-Dglucopyranoside and methyl $\alpha$-D-galactopyranoside, respectively. $\mathrm{Bu}_{3} \mathrm{SnH}$-mediated aryl radical cyclization of $\mathbf{3}$ provided exclusively the hydrogenolysis product $\mathbf{1 2}$. The reaction of $\mathbf{4}$ gave the reduced uncyclized product $\mathbf{1 3}$ and only traces of $\mathbf{4 A}$, resulting from 11-endo aryl radical cyclization. In previous papers we described that in similar $\mathrm{Bu}_{3} \mathrm{SnH}$-mediated radical reaction of orthoiodoallyloxybenzamides, analogs of $\mathbf{3}$ and $\mathbf{4}$, we obtained macrolactams resulting from 11-endo cyclization. An hypothesis to explain the differences is presented. It was assumed that in the aryl radical formed from iodobenzamides there is a suitable conformation to cyclization, which is stabilized by an intramolecular hydrogen bond.
\end{abstract}

Keywords: aryl radical cyclization; 11-membered macrocycles; 2-iodobenzoates.

\section{INTRODUÇÃO}

Na química orgânica moderna, reações de carbociclização radicalar adquiriram um importante papel na síntese de heterociclos ${ }^{1-5}$. Reações de ciclização radicalar têm sido extensivamente investigadas nos últimos anos e o método mais usado é aquele em que se utiliza o hidreto de tri- $n$-butilestanho ${ }^{6}$. Um produto cíclico reduzido é formado via mecanismo radicalar a partir de um precursor contendo halogênio e insaturação. Nestas reações o $\mathrm{Bu}_{3} \mathrm{SnH}$ atua como gerador de um radical inicial no precursor, por meio da quebra homolítica da ligação carbono halogênio, e como redutor do radical cíclico ${ }^{7}$

Halobenzenos substituídos em posição orto com grupos alquenila têm sido amplamente utilizados para síntese de compostos contendo ciclos fundidos a anéis aromáticos ${ }^{8-26}$. São obtidos os produtos provenientes das ciclizações dos modos endo e/ou exo e os de hidrogenólise (Figura 1).

Encontram-se registradas na literatura a dificuldade ou mesmo a impossibilidade de se obter, por ciclização radicalar mediada por $\mathrm{Bu}_{3} \mathrm{SnH}$, macrociclos denominados "menores" (10 a 12 membros), ao contrário do que se observa no caso de macrociclos "maiores"(14 a 16 membros $)^{27,28}$. É conhecido também que a formação de radicais arila a partir de orto-halobenzamidas contendo hidrogênio ligado a carbono saturado em posição 5 em relação ao radical, pode ser seguida de transferência do hidrogênio do carbono saturado para o grupo arila, com a conseqüente formação de um radical $\alpha$ amidoíla (Figura 2). O novo radical formado pela transferência radicalar 1,5 pode conduzir ao correspondente produto de hidrogenólise e, dependendo da estrutura do substrato, a produtos de ciclização envolvendo o radical $N$-amidoíla ${ }^{29}$.

A despeito de os dados da literatura revelarem aspectos negativos da síntese de macrociclos "menores" e da ciclização de radicais arila em substratos contendo hidrogênio ligado a carbono saturado $\alpha$-amidó́lico, decidimos utilizar reações de carbociclização radicalar mediadas por $\mathrm{Bu}_{3} \mathrm{SnH}$ para sintetizar as benzomacrolactamas $\mathbf{1 A}$ e/ou $\mathbf{1 B}$ e $\mathbf{2 A}$ e/ou $\mathbf{2 B}$ a partir das orto-

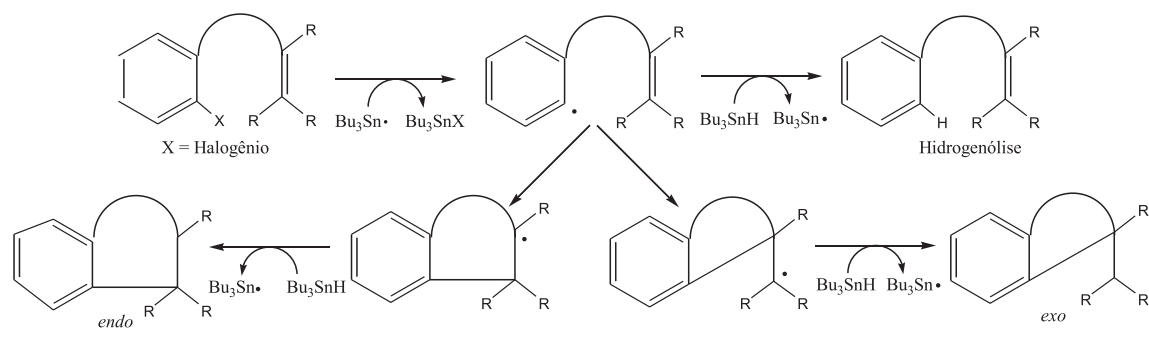

Figura 1. Produtos de reação de orto-haloalquenilbenzenos com $\mathrm{Bu}_{3} \mathrm{SnH}$ 
<smiles>[R]N(C(=O)c1ccccc1)C([2H])([2H])C([2H])([2H])N([R])C(=O)c1ccccc1</smiles>

Figura 2. Mecanismo da transferência de radical 1,5

aliloxiiodobenzamidas 1 e $\mathbf{2}$, respectivamente (Figura 3). Tínhamos a expectativa de que a presença de unidades sacarídicas conferisse aos substratos conformações adequadas para a ciclização e que a presença do oxigênio do grupo aliloxila nas cadeias laterais dos substratos ( $\mathbf{1}$ e $\mathbf{2})$ tornasse as ciclizações mais rápidas, em relação às transferências 1,5 e às reduções dos radicais arila. Esta última hipótese baseia-se no relato de que a velocidade de reações de ciclização de precursores contendo oxigênio na cadeia lateral é muito maior que a de seus análogos de carbono ${ }^{30-33}$.

As orto-iodobenzamidas $\mathbf{1}$ e $\mathbf{2}$ submetidas à reação com $\mathrm{Bu}_{3} \mathrm{SnH}$ conduziram às respectivas benzomacrolactamas $\mathbf{1 A}$ e $\mathbf{2 A}$ (Figura 3), provenientes de ciclização 11-endo, com rendimentos de $40 \mathrm{e}$ $35 \%$, respectivamente ${ }^{33,34}$. Uma macrolactama formada por ciclização 11-endo foi obtida, com rendimento de $14 \%$, por reação radicalar do substrato $N$-(3-aliloxipropil)-2-iodobenzamida, que não possui a unidade sacarídica ${ }^{33}$. Estes resultados indicaram que nossa hipótese de que a restrição conformacional imposta pela presença da unidade sacarídica deveria estar correta e que a estereoquímica de C-4 do anel piranosídico não influenciava no modo de ciclização e nem, de forma significativa, nos rendimentos dos produtos ciclizados $^{33,34}$.

Tendo em vista o sucesso obtido nas reações de ciclização das iodobenzamidas $\mathbf{1}$ e 2, decidimos utilizar a reação de carbociclização radicalar mediada por $\mathrm{Bu}_{3} \mathrm{SnH}$ para sintetizar as benzomacrolactonas $\mathbf{3 A}$ e/ou $\mathbf{3 B}$ e $\mathbf{4 A}$ e/ou $\mathbf{4 B}$ a partir dos orto-iodoaliloxibenzoatos $\mathbf{3}$ e $\mathbf{4}$, respectivamente (Figura 3 ).

\section{RESULTADOS E DISCUSSÃO}

Os orto-iodoaliloxibenzoatos 4- $O$-alil-2,3-di- $O$-benzil-6- $O$-(2iodobenzoil)- $\alpha$-D-glicopiranosídeo de metila (3) e 4- $O$-alil-2,3-di$O$-benzil-6- $O$-(2-iodobenzoil)- $\alpha$-D-galactopiranosídeo de metila (4) foram obtidos a partir de $\alpha$-D-glicopiranosídeo de metila (5) e $\alpha$ D-galactopiranosídeo de metila (5'), respectivamente, em sete etapas (Figura 4), utilizando-se reações clássicas da química de carboidratos. Protegeram-se as hidroxilas de C-4 e C-6 na forma de acetal benzilidênico, as hidroxilas de C-2 e C-3 foram benziladas, o grupo acetal benzilidênico foi removido e a hidroxila de C-6 foi protegida de forma regiosseletiva como éter de tert-butildimetilsilila. Em seguida, a hidroxila de C-4 foi alilada e o grupo protetor da hidroxila de C-6 foi removido. Posteriormente, por reação de $\mathbf{1 1}$ e 11' com cloreto de 2-iodobenzoíla foram obtidas, respectivamente, os orto-iodoaliloxibenzoatos 3 e 4.

Inicialmente, as reações de ciclização radicalar foram desenvolvidas utilizando-se as condições preconizadas para se minimizar a formação dos produtos de hidrogenólise e a ocorrência de reações intermoleculares, ou seja, alta diluição e adição lenta da solução de $\mathrm{Bu}_{3} \mathrm{SnH}$ e quantidade catalítica de AIBN em benzeno, sobre as soluções dos substratos ( 3 ou 4) em benzeno, sob refluxo ${ }^{28,35,36}$.

Após eliminação do solvente, os resíduos obtidos foram submetidos à separação dos produtos em cromatografia em coluna de sílica. Da reação desenvolvida com o substrato 3 foi possível isolar apenas um produto, caracterizado pelos espectros de RMN como sendo o 4- $O$-alil-2,3-di- $O$-benzil-6- $O$-benzoil- $\alpha$-D-glicopiranosídeo de metila (12). Da reação radicalar desenvolvida com $500 \mathrm{mg}(0,77$ mmol) de 4 isolaram-se o 4- $O$-alil-2,3-di- $O$-benzil-6- $O$-benzoil$\alpha$-D-galactopiranosídeo de metila (13) e apenas $30 \mathrm{mg}$ de uma mistura, que não foi possível purificar. No entanto, nos espectros de RMN uni e bidimensionais $\left({ }^{1} \mathrm{H}\right.$, de ${ }^{13} \mathrm{C}$, DEPT, COSY e HMQC) da mistura observam-se sinais e manchas de correlação que indicam a presença da macrolactona $\mathbf{4 A}$, proveniente da ciclização 11-endo (Figura 5).
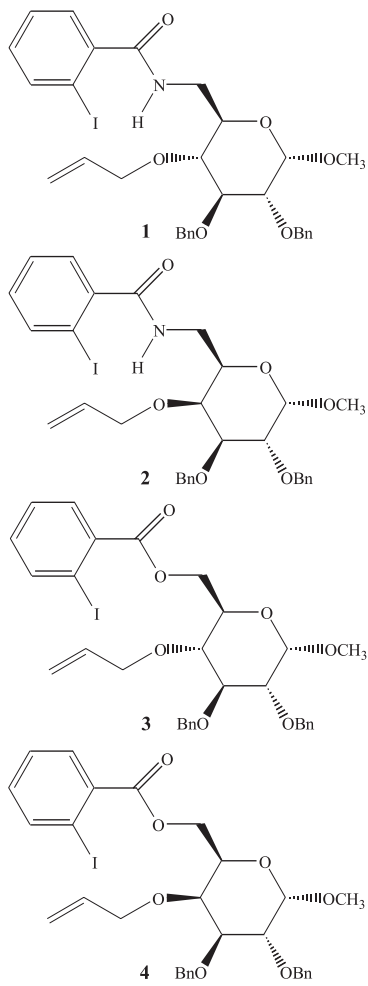
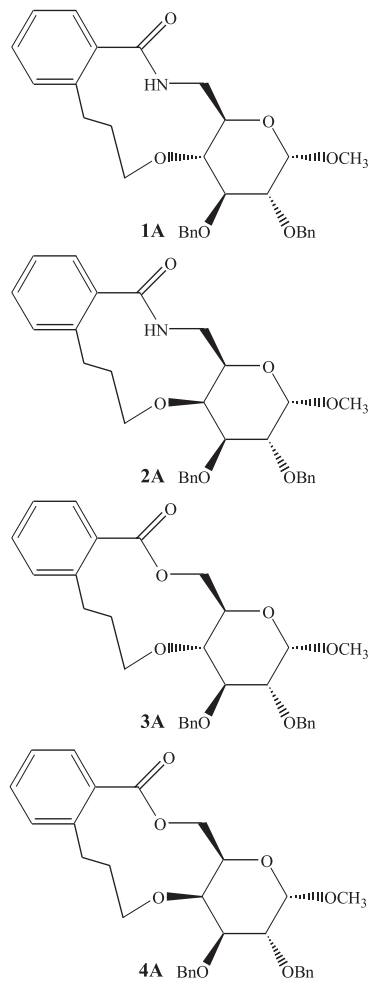
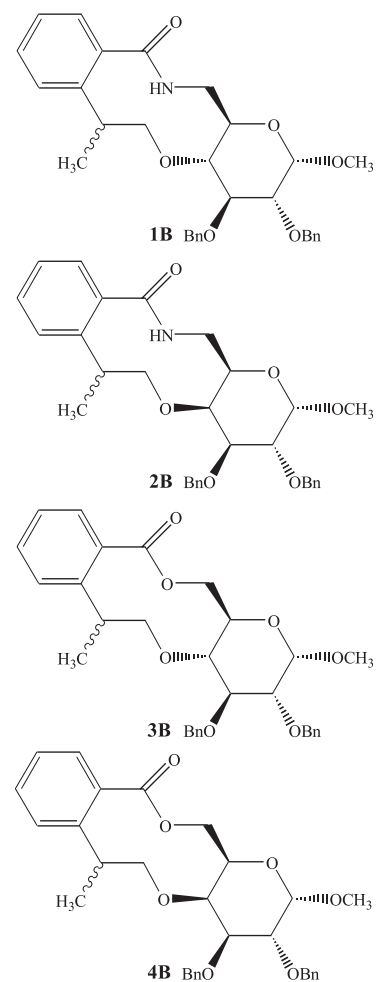

Figura 3. Estruturas das benzamidas, benzoatos e seus possíveis produtos de ciclização 

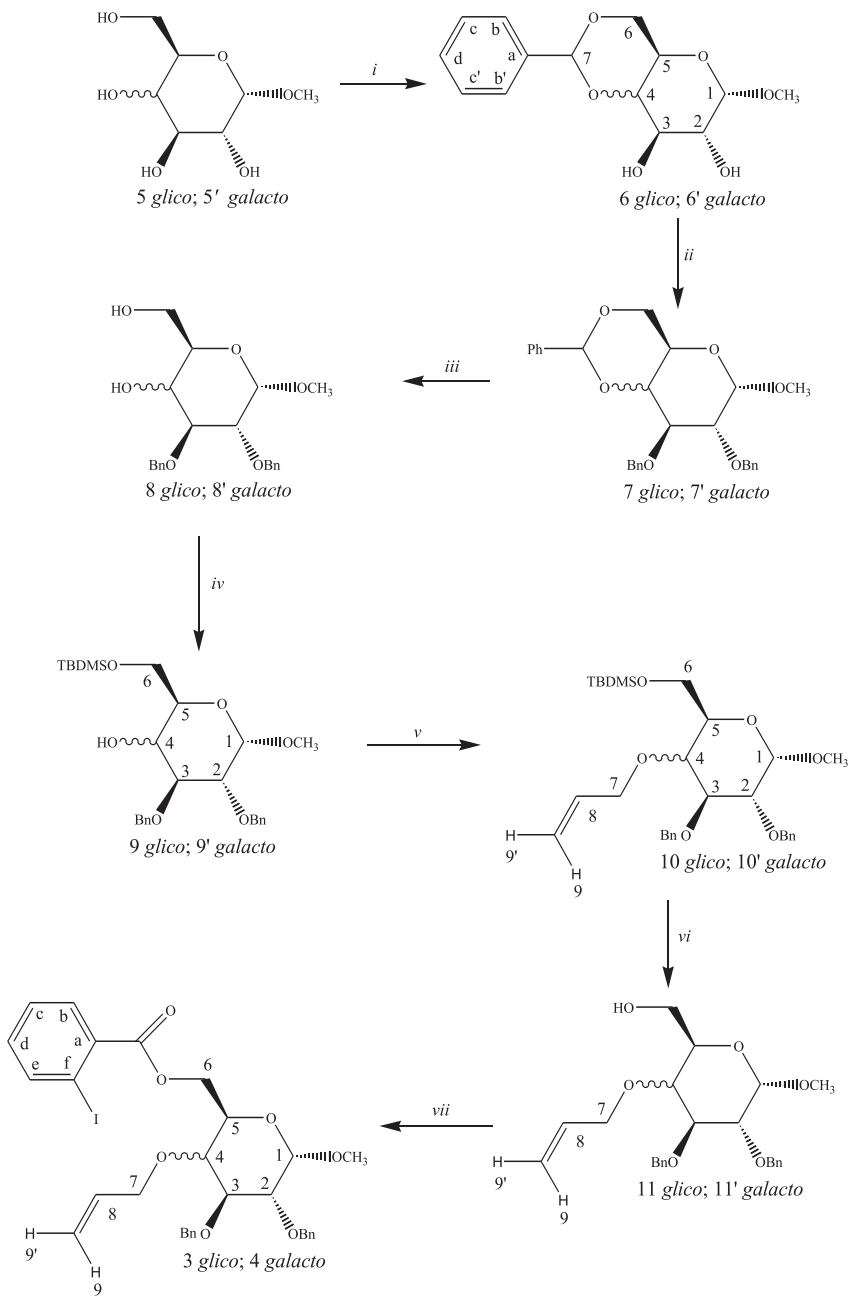

Reagentes e condições: i) benzaldeido, cloreto de zinco, $25^{\circ} \mathrm{C}$; ii) brometo de benzila, brometo de tetrabutilamônio, diclorometano, hidróxido de sódio $50 \% \mathrm{p} / \mathrm{v}, 25{ }^{\circ} \mathrm{C}$ (para obtenção de 6), cloreto de benzila, $\mathrm{KOH}$, refluxo (para obtenção de 6'); iii) $\mathrm{HCl}$, acetona, água, refluxo; iv) cloreto de tertbutildimetilsilila, trietilamina, 4-N-dimetilaminopiridina, $25{ }^{\circ} \mathrm{C}$; v) brometo de alila, brometo de tetrabutilamônio, diclorometano, hidróxido de sódio $50 \%$ $\mathrm{p} / \mathrm{v}, 25^{\circ} \mathrm{C}$; vi) TBFA, THF anidro, $25^{\circ} \mathrm{C} e$, vii) cloreto de 2-iodobenzoila, trietilamina, diclorometano anidro, $25{ }^{\circ} \mathrm{C}$

\section{Figura 4. Rota de síntese dos orto-iodoaliloxibenzoatos 3 e 4}

Tendo em vista o insucesso na obtenção dos macrociclos desejados, desenvolveu-se a reação do substrato $\mathbf{3}$ utilizado-se o hexabutildiestanila como agente de ciclização, seguindo-se metodologia descrita na literatura ${ }^{37,38}$. Também neste caso não se obteve o macrociclo e sim, o produto de hidrogenólise $\mathbf{1 2}$.

É possível propor uma explicação para as diferenças das reações de ciclização radicalar das orto-iodoaliloxibenzamidas (1 e 2) e dos correspondentes orto-iodoaliloxibenzoatos (3 e 4).

Nas orto-halobenzamidas, além da restrição de rotação da ligação C-N, característica das amidas, há restrição da rotação da ligação $\mathrm{Ph}-\mathrm{C}$, atribuída ao efeito estérico do halogênio em orto, com predominância do rotâmero no qual o anel aromático e a carbonila não se apresentam coplanares ${ }^{39}$. No que se refere às possíveis conformações relativas à ligação $\mathrm{C}-\mathrm{N}$, predomina o confôrmero no qual o grupo mais volumoso ligado ao nitrogênio se encontra syn em relação ao oxigênio carbonílico ${ }^{39}$. Portanto, no caso da benzamida $\mathbf{1}$, por ex., predominaria o confôrmero $\mathbf{1} a$ (Figura 6). O tempo de vida de radicais arila não excede $10^{-5} \mathrm{~s}$, sendo muito menor que o tempo
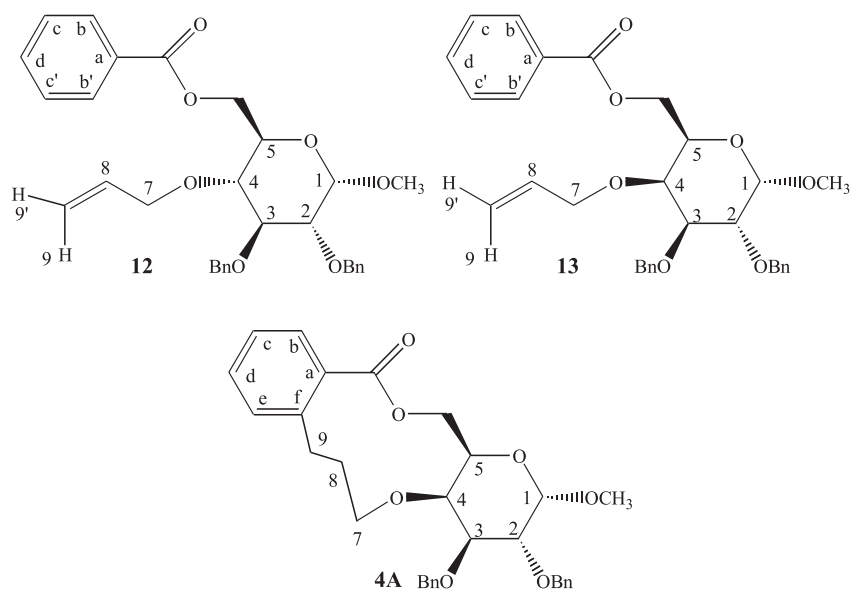

Figura 5. Produtos isolados das reações de $\mathbf{3}$ e $\mathbf{4} \mathrm{com} \mathrm{Bu}_{3} \mathrm{SnH}$

gasto para interconversão dos rotâmeros syn e anti de amidas ${ }^{29}$, o que suporta a hipótese de que não há rotação da ligação C-N durante a existência dos radicais arila. Assumindo que as reatividades dos rotâmeros $\mathbf{1} \boldsymbol{a} / \mathbf{1} \boldsymbol{b}$ da benzamida $\mathbf{1}$ seriam aproximadamente iguais frente ao radical tributilestanila, as quantidades relativas dos radicais formados por abstração de um átomo de iodo seriam determinadas pelas concentrações dos rotâmeros, portanto, seria formado predominantemente o radical 1 $\boldsymbol{a}^{\prime}$ (Figura 6). Em relação à ligação C6C5, no rotâmero $\mathbf{1} \boldsymbol{a}$ ' há três conformações alternadas, representadas na projeção de Newman (Figura 6). Em uma das conformações (1 $a$ ") há possibilidade de estabilização por formação de ligação de hidrogênio entre o hidrogênio ligado ao nitrogênio e o oxigênio do grupo aliloxila, formando-se um ciclo de seis membros (Figura 6). Possivelmente, esta seja a conformação adequada para que ocorra o ataque do radical arila à dupla ligação, com formação subseqüiente do produto ciclizado.

Ao contrário do que se pode prever nas benzamidas, nos benzoésteres $\mathbf{3}$ e $\mathbf{4}$ por não haver restrição de rotação da ligação $\mathrm{C}-\mathrm{O}$ e nem estabilização de nenhuma das conformações em rela-

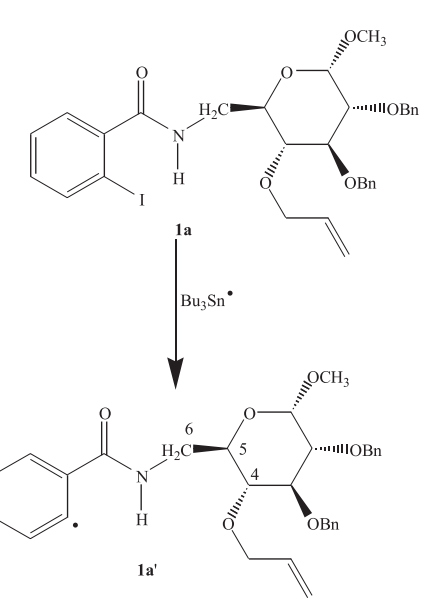<smiles>C=CCO[C@H]1[C@@H](CNC(=O)c2ccccc2I)O[C@H](OC)[C@@H]([Pb])[C@@H]1OCc1ccccc1</smiles><smiles>CC1C2CC(OO2)C(NC(=O)c2ccccc2)C1NC(=O)c1ccccc1</smiles>

Figura 6. Conformações da orto-iodoaliloxibenzamida 1 e do radical correspondente 
ção à ligação C6-C5, um grande número de confôrmeros dos radicais arila deve coexistir. Assim, a probabilidade de ocorrência de radicais arila cuja conformação seja adequada para o ataque à dupla ligação é muito pequena, o que pode ser a justificativa para não se ter indício de formação de produto ciclizado a partir de $\mathbf{3}$ e de que da reação de $\mathbf{4}$ com $\mathrm{Bu}_{3} \mathrm{SnH}$ obteve-se pequena quantidade de uma mistura na qual, possivelmente, há um produto proveniente de ciclização 11-endo (4A).

A hipótese de que a presença de ligação de hidrogênio intramolecular no radical arila é um fator importante para que haja formação de produtos de ciclização é corroborada pelos resultados obtidos nas reações radicalares mediadas por $\mathrm{Bu}_{3} \mathrm{SnH}$, realizadas com as amidas secundárias 14 e 16 e as correspondentes benzamidas $\mathrm{N}$-benziladas 15 e 17. As orto-iodobenzamidas 14 e 16, em que há possibilidade de formação de ligação de hidrogênio intramolecular, como proposto para o radical $\mathbf{1} \boldsymbol{a}$ " (Figura 4), levaram às lactamas provenientes de ciclização 11 -endo ${ }^{33}$ e 12 -endo $o^{40}$, respectivamente. Por outro lado, as amidas terciárias (15 e 17) conduziram apenas aos correspondentes produtos de hidrogenólise ${ }^{41}$.
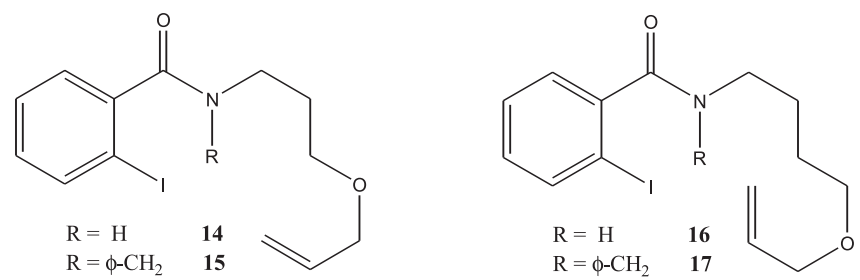

\section{PARTE EXPERIMENTAL}

\section{Procedimentos gerais}

As temperaturas de fusão foram determinadas em um aparelho Mettler FP82HT e não foram corrigidas. Os poderes rotatórios específicos, $[\alpha]_{\mathrm{D}}$, foram medidos em polarímetro Perkin-Elmer 341 a $25{ }^{\circ} \mathrm{C}$. Os espectros de $\mathrm{RMN}{ }^{1} \mathrm{H}$ e ${ }^{13} \mathrm{C}$ foram registrados nos espectrômetros Bruker Avance DPX 200 ou DRX 400. Como referência interna foi utilizado o tetrametilsilano. Os espectros no infravermelho foram registrados em aparelho Mattson Instruments Galaxy 3000. As análises elementares foram realizadas em aparelho Perkin-Elmer $2400 \mathrm{CHN}$. As cromatografias em coluna foram realizadas com sílica gel 60, 70-230 mesh (Merck). O termo "elaboração usual" significa que as fases orgânicas foram reunidas, lavou-se com água destilada, manteve-se a fase orgânica em contato com sulfato de sódio anidro durante cerca de $15 \mathrm{~min}$, filtrou-se e o solvente foi removido sob pressão reduzida em evaporador rotatório. Os procedimentos de síntese e os dados físico-químicos dos intermediários 6, 6', 7, 7', 8 e 8' encontram-se descritos na literatura ${ }^{42-46}$. Os procedimentos seguidos para sínteses das outras substâncias foram adaptados e as referências utilizadas encontram-se relacionadas após o nome da substância cuja metodologia de síntese é descrita.

\section{2,3-di- $O$-benzil-6-O-tert-butildimetilsilil- $\alpha$-D- galactopiranosídeo de metila $\left(9^{\prime}\right)^{47}$}

A uma solução de 2,3-di- $O$-benzil- $\alpha$-D-galactopiranosídeo de metila (8') (2,00 g, 5,34 mmol) em diclorometano $(20 \mathrm{~mL})$, sob agitação e banho de gelo, foram adicionados trietilamina $(7,5 \mathrm{~mL})$, cloreto de tert-butildimetilsilila $(870 \mathrm{mg}, 5,8 \mathrm{mmol})$ e $4-N$ dimetilaminopiridina (20 mg, 0,16 mmol). O sistema foi vedado e mantido sob agitação magnética, à temperatura ambiente, por $12 \mathrm{~h}$. Adicionou-se solução aquosa de $\mathrm{HCl} 3 \mathrm{~mol} / \mathrm{L}$, separou-se a fase or- gânica, extraiu-se a fase aquosa com diclorometano e seguiu-se o procedimento de "elaboração usual". O resíduo obtido foi submetido à purificação em CCS e o produto foi eluído da coluna com hexano/ acetato 8:2. Foram obtidos 2,00 g (4,13 mmol, 78\%) de 2,3-di- $O$ benzil-6-O-tert-butildimetilsilil- $\alpha$-D-galactopiranosídeo de metila (9'). Óleo incolor. $[\alpha]_{\mathrm{D}}+6,8\left(c 1,00, \mathrm{CHCl}_{3}\right)$. IV $\left(\mathrm{KBr}, \mathrm{cm}^{-1}\right) v_{\text {max }}$ : 3500, 3100, 3050, 2900, 2850, 1500, 1450, 1100, 1050, 750, 700. RMN ${ }^{1} \mathbf{H}\left(200 \mathrm{MHz}, \mathrm{CDCl}_{3}\right.$ ) $\delta: ~ 7,30-7,23$ (m, 10 H-aromáticos), $4,75\left(\mathrm{dl}, J_{\text {gem }}=11,5 \mathrm{~Hz}, 2 \mathrm{H}\right.$-benzílicos), 4,64 (d, $J_{\text {gem }}=11,9 \mathrm{~Hz}, \mathrm{H}-$ benzílico), 4,60 (d, $J_{\mathrm{gem}}=11,9 \mathrm{~Hz}, \mathrm{H}$-benzílico), 4,60 (d, $J_{1,2}=2,8 \mathrm{~Hz}$, H-1), 3,97-3,96 (m, H-4), 3,82-3,75 (m, H-2, H-3, H-6), 3,71-3,75 (m, H-5, H-6'), 3,30 (s, $\left.\mathrm{CH}_{3} \mathrm{O}\right), 2,56$ (sl, $\left.\mathrm{OH}\right), 0,82$ (s, $\left.\mathrm{C}\left(\mathrm{CH}_{3}\right)_{3}\right)$, $0,01\left(\mathrm{~s}, \mathrm{Si}\left(\mathrm{CH}_{3}\right)_{2}\right)$. RMN ${ }^{13} \mathbf{C}\left(50 \mathrm{MHz}, \mathrm{CDCl}_{3}\right) \delta: 138,33,138,18(\mathrm{C}$ ipso), 128,39, 128,28, 127,95, 127,72, 127,67 (C-aromáticos), 98,41 (C-1), 77,73 (C-3), 75,74, (C-2), 73,42, 72,67 (C-benzílicos), 69,63 (C-5), 67,64 (C-4), 62,50 (C-6), 55,04 ( $\left.\mathrm{CH}_{3} \mathrm{O}\right), 25,78\left(\mathrm{C}\left(\mathrm{CH}_{3}\right)_{3}\right), 18,19$ $(C-\mathrm{Si}),-5,49$ e $-5,55\left(\mathrm{Si}\left(\mathrm{CH}_{3}\right)_{2}\right)$. Análise elementar: encontrado: $C$ $66,69 \%, H 7,65 \%$; requerido para $\mathrm{C}_{27} \mathrm{H}_{40} \mathrm{O}_{6} \mathrm{Si}: C 66,36 \%, H 8,25 \%$.

\section{2,3-di-O-benzil-6-O-tert-butildimetilsilil- $\alpha$-D-glicopiranosídeo de metila $(9)^{47}$}

O tratamento do 2,3-di- $O$-benzil- $\alpha$-D-glicopiranosídeo de metila (8) (1,36 g, 3,60 mmol) com cloreto de tert-butildimetilsilila, nas mesmas condições descritas para o composto $\mathbf{8}$, , conduziu ao produto $9(1,67 \mathrm{~g}, 3,43 \mathrm{mmol}, 95 \%)$ como um óleo incolor. $[\alpha]_{\mathrm{D}}$ $+21,8\left(c\right.$ 3,96, $\left.\mathrm{CHCl}_{3}\right)$. IV $\left(\mathrm{KBr}, \mathrm{cm}^{-1}\right) v_{\mathrm{max}}: 3600,3100,3050,2920$, 2870, 1490, 1450, 1100, 1050, 750, 700. RMN ${ }^{1} \mathbf{H}(200 \mathrm{MHz}$, $\left.\mathrm{CDCl}_{3}\right) \delta:$ 7,40-6,97 (m, $10 \mathrm{H}$-aromáticos), 5,00 (d, $J_{\text {sem }}=11,4 \mathrm{~Hz}$, H-benzílico), 4,79 (d, $J_{\text {gem }}=12,1 \mathrm{~Hz}, \mathrm{H}$-benzílico), 4,78 (d, $J_{\text {gem }}=$ 11,4 Hz, H-benzílico), 4,67 (d, $J_{\text {gem }}=12,1 \mathrm{~Hz}, \mathrm{H}$-benzílico), 4,63 $\left(\mathrm{d}, J_{1,2}=3,5 \mathrm{~Hz}, \mathrm{H}-1\right), 3,87-3,75$ e 3,65-3,49 (2 m, H-3, H-4, H-5, 2 H-6), 3,51 (dd, $\left.J_{23}=9,6 \mathrm{~Hz}, J_{2,1}=3,5 \mathrm{~Hz}, \mathrm{H}-2\right), 3,39\left(\mathrm{~s}, \mathrm{CH}_{3} \mathrm{O}\right)$, 2,18 (sl, OH), 0,90 (s, $\left.\mathrm{C}\left(\mathrm{CH}_{3}\right)_{3}\right), 0,80\left(\mathrm{~s}, \mathrm{Si}\left(\mathrm{CH}_{3}\right)_{2}\right) . \mathbf{R M N}{ }^{13} \mathbf{C}(50$ $\mathrm{MHz}, \mathrm{CDCl}_{3}$ ) $\delta: 139,05,138,33$ (C ipso), 128,76, 128,67, 128,31, 128,25, 128,12, 128,01 (C-aromáticos), 98,23 (C-1), 80,70, 71,79, 70,97 (C-3, C-4, C-5), 79,81 (C-2), 76,62, 75,72 (C-benzílicos), 63,93 (C-6), 55,29 ( $\left.\mathrm{CH}_{3} \mathrm{O}\right), 26,14\left(\mathrm{C}\left(\mathrm{CH}_{3}\right)_{3}\right), 18,59(\mathrm{C}-\mathrm{Si}),-5,17$ e -5,21 $\left(\mathrm{Si}\left(\mathrm{CH}_{3}\right)_{2}\right)$. Análise elementar: encontrado: $\mathrm{C} \mathrm{66,27 \% ,H}$ $8,72 \%$; requerido para $\mathrm{C}_{27} \mathrm{H}_{40} \mathrm{O}_{6} \mathrm{Si}: \mathrm{C} 66,36 \%, H 8,25 \%$.

\section{4-O-alil-2,3-di-O-benzil-6-O-tert-butildimetilsilil- $\alpha$-D- galactopiranosídeo de metila $\left(\mathbf{1 0}^{\prime}\right)^{48}$}

A uma mistura de 2,3-di-O-benzil-6-O-tert-butildimetilsilil- $\alpha$ D-galacto-piranosídeo de metila (9') (200 mg, 0,41 mmol), brometo de tetrabutilamônio (64 mg, 0,20 mmol), diclorometano $(3 \mathrm{~mL})$ e solução de hidróxido de sódio a $50 \%$ p/v $(9 \mathrm{~mL})$, foi adicionado, sob agitação magnética e banho de gelo, brometo de alila $(0,12$ $\mathrm{mL}, 0,17 \mathrm{mg}, 1,40 \mathrm{mmol})$. A mistura foi deixada sob agitação, à temperatura ambiente, por $24 \mathrm{~h}$. Separou-se a fase orgânica, extraiu-se a fase aquosa com diclorometano $(3 \times 10 \mathrm{~mL})$ e seguiu-se o procedimento de "elaboração usual". O resíduo obtido foi submetido à purificação em CCS (hexano/acetato 9:1), obtendo-se 2,00 g (4,13 mmol, 67\%) de 4-O-alil-2,3-di-O-benzil-6-O-tertbutildimetilsilil- $\alpha$-D-galactopiranosídeo de metila (10'). Sólido amorfo. $[\alpha]_{\mathrm{D}}+101,7\left(c 1,00, \mathrm{CHCl}_{3}\right)$. IV $\left(\mathrm{KBr}, \mathrm{cm}^{-1}\right) \mathrm{v}_{\max }: 3350$, 3100, 3050, 2900, 2850, 1500, 1450, 1250, 1050, 850, 750, 700. RMN ${ }^{1} \mathbf{H}\left(200 \mathrm{MHz}, \mathrm{CDCl}_{3}\right) \delta:$ 7,38-7,27 (m, $10 \mathrm{H}$-aromáticos), $5,94\left(\mathrm{dtd}, J_{8,9}=17,2 \mathrm{~Hz}, J_{8,9}=10,2 \mathrm{~Hz}, J_{8,7}=J_{8,7}=5,8 \mathrm{~Hz}, \mathrm{H}-8\right)$, 5,21 (ddd, $J_{9,8}=17,2 \mathrm{~Hz}, J_{\mathrm{gem}}=3,3 \mathrm{~Hz}, J_{9,7}=1,3 \mathrm{~Hz}, \mathrm{H}-9$ ), 5,12 (ddd, $\left.J_{9,8}=10,2 \mathrm{~Hz}, J_{\text {gem }}=3,3 \mathrm{~Hz}, J_{9^{\prime}, 7}=1,3 \mathrm{~Hz}, \mathrm{H}-9^{\prime}\right), 4,83\left(\mathrm{~d}, J_{\text {gem }}=11,4\right.$ Hz, H-benzílico), 4,78 (d, $J_{\text {gem }}=11,4 \mathrm{~Hz}, \mathrm{H}$-benzílico), 4,72 (d, 
$J_{\text {gem }}=12,2 \mathrm{~Hz}, \mathrm{H}$-benzílico), 4,66 (d, $J_{\text {gem }}=12,2 \mathrm{~Hz}, \mathrm{H}$-benzílico), $4,65\left(\mathrm{~d}, J_{1,2}=3,4 \mathrm{~Hz}, \mathrm{H}-1\right), 4,62\left(\mathrm{dtd}, J_{\text {gem }}=12,5 \mathrm{~Hz}, J_{7,8}=5,8 \mathrm{~Hz}\right.$, $\left.J_{79}=J_{79}=1,3 \mathrm{~Hz}, \mathrm{H}-7\right), 4,41\left(\mathrm{dtd}, J_{\text {gem }}=12,5 \mathrm{~Hz}, J_{7,8}=5,8 \mathrm{~Hz}, J_{7,9}=\right.$ $J_{7^{\prime}, 9^{\prime}}=1,3 \mathrm{~Hz}, \mathrm{H}-7$ ') $, 4,02$ (dd, $\left.J_{2,3}=9,6 \mathrm{~Hz}, J_{2,1}=3,4 \mathrm{~Hz}, \mathrm{H}-2\right), 3,86$ (m, 2 H-6), 3,77-3,59 (m, H-3, H-4, H5), 3,36 (s, OCH ) $^{2}$ 0,88 (s, $\left.\mathrm{C}\left(\mathrm{CH}_{3}\right)_{3}\right), 0,41\left(\mathrm{~s}, \mathrm{Si}\left(\mathrm{CH}_{3}\right)_{2}\right) . \mathbf{R M N}{ }^{13} \mathbf{C}\left(50 \mathrm{MHz}, \mathrm{CDCl}_{3}\right) \delta: 138,80$, 138,57 (C ipso), 135,51 (C-8), 128,26, 127,96, 127,56, 127,44 (Caromáticos), 116,62 (C-9), 98,74 (C-1), 78,94, 74,61, 70,75 (C-3, C-4, C-5), 76,57 (C-2), 73,90 (C-7), 73,53, 73,09 (C-benzílicos), $61,42(\mathrm{C}-6), 55,16\left(\mathrm{OCH}_{3}\right), 25,80\left(\mathrm{C}\left(\mathrm{CH}_{3}\right)_{3}\right), 18,16(\mathrm{C}-\mathrm{Si}),-5,47$ e -5,54 $\left(\mathrm{Si}\left(\mathrm{CH}_{3}\right)_{2}\right)$. Análise elementar: encontrado: $C$ 67,81\%, H $8,63 \%$; requerido para $\mathrm{C}_{30} \mathrm{H}_{44} \mathrm{O}_{6} \mathrm{Si}: C 68,15 \%, H 8,39 \%$.

\section{4-O-alil-2,3-di-O-benzil-6-O-tert-butildimetilsilil- $\alpha$-D- glicopiranosídeo de metila $(\mathbf{1 0})^{48}$}

Submetendo-se o derivado 9 (0,42 $\mathrm{g}, 0,86 \mathrm{mmol})$ às mesmas condições descritas para o derivado galacto 9' obteve-se $0,32 \mathrm{~g}$ $(0,61 \mathrm{mmol}, 70 \%)$ de 4-O-alil-2,3-di-O-benzil-6-O-tertbutildimetilsilil- $\alpha-\mathrm{D}$-glicopiranosídeo de metila (10). Óleo incolor. $[\alpha]_{\mathrm{D}}+67,3\left(c 1,11, \mathrm{CHCl}_{3}\right) . \mathbf{I V}\left(\mathrm{KBr}, \mathrm{cm}^{-1}\right) \mathrm{v}_{\max }: 3350,2900$, 2850, 1600, 1580, 1500, 1450, 1270, 1050, 850, 700. RMN ${ }^{1} \mathbf{H}$ $\left(200 \mathrm{MHz}, \mathrm{CDCl}_{3}\right) \delta:$ 7,37-7,30 (m, $10 \mathrm{H}$-aromáticos), 6,03-5,83 (m, H-8), 5,26 (dd, $\left.J_{9,8}=17,2 \mathrm{~Hz}, J=1,5 \mathrm{~Hz}, \mathrm{H}-9\right), 5,16$ (d, $J_{9,8}=$ $11,5 \mathrm{~Hz}, \mathrm{H}-9$ '), 4,97-4,63 (m, 4 H'-benzílicos), 4,61 (d, $J_{1,2}=3,5$ $\mathrm{Hz}, \mathrm{H}-1), 4,35$ (dd, $J_{\text {gem }}=12,3 \mathrm{~Hz}, J_{7,8}=5,6 \mathrm{~Hz}, \mathrm{H}-7$ ), 4,14 (dd, $J_{\text {gem }}=12,3 \mathrm{~Hz}, J_{7,8}=5,6 \mathrm{~Hz}, \mathrm{H}-7$ '), 3,94 (t, $\left.J_{3,4}=J_{3,2}=9,2 \mathrm{~Hz}, \mathrm{H}-3\right)$, $3,81\left(\mathrm{~d}, J_{6,5}=2,9 \mathrm{~Hz}, 2 \mathrm{H}-6\right), 3,60\left(\mathrm{td}, J_{5,4}=9,2 \mathrm{~Hz}, J_{5,6}=2,9 \mathrm{~Hz}, \mathrm{H}-\right.$ 5), 3,47 (dd, $\left.J_{23}=9,2 \mathrm{~Hz}, J_{2,}=3,5 \mathrm{~Hz}, \mathrm{H}-2\right), 3,44-3,35$ (m, H-4), $3,38\left(\mathrm{~s}, \mathrm{OCH}_{3}\right), 0,90\left(\mathrm{~s}, \mathrm{C}\left(\mathrm{CH}_{3}\right)_{3}\right), 0,61\left(\mathrm{~s}, \mathrm{Si}\left(\mathrm{CH}_{3}\right)_{2}\right) . \mathbf{R M N}{ }^{13} \mathbf{C}(50$ $\mathrm{MHz}, \mathrm{CDCl}_{3}$ ) $\delta: 139,01,138,49$ (C ipso), 135,22 (C-8), 128,62, 128,36, 128,25, 128,04, 127,84 (C-aromáticos), 116,87 (C-9), 98,13 (C-1), 82,29 (C-3), 80,29 (C-2), 77,70 (C-4), 71,73 (C-5), 76,04 (C-7), 73,98, 73,57 (C-benzílicos), 62,40 (C-6), 55,13 (OCH $), 26,14$ $\left(\mathrm{C}\left(\mathrm{CH}_{3}\right)_{3}\right), 18,54(\mathrm{C}-\mathrm{Si}),-4,97$ e $-5,18\left(\mathrm{Si}\left(\mathrm{CH}_{3}\right)_{2}\right)$. Análise elementar: encontrado: $C 68,57 \%, H 8,74 \%$; requerido para $\mathrm{C}_{30} \mathrm{H}_{44} \mathrm{O}_{6} \mathrm{Si}$ : C $68,15 \%, H 8,39 \%$.

\section{4- $O$-alil-2,3-di- $O$-benzil- $\alpha$-D-galactopiranosídeo de metila $(11 ')^{49}$}

A uma solução do 4- $O$-alil-6- $O$-tert-butildimetilsilil-2,3-di- $O$ benzil- $\alpha$-D-galactopiranosídeo de metila (10') (300 mg, 0,57 mmol) em de THF anidro $(3,3 \mathrm{~mL})$ foi adicionado, a $0{ }^{\circ} \mathrm{C}$, TBFA $(530 \mathrm{mg}$, $1,63 \mathrm{mmol}$ ). Após atingir a temperatura ambiente, a mistura foi mantida sob agitação por mais $2 \mathrm{~h}$. Ao se observar o término da reação, destilou-se o solvente e adicionaram-se $20 \mathrm{~mL}$ de água ao resíduo. Em seguida, procedeu-se à extração da fase aquosa com diclorometano (3 x $40 \mathrm{~mL})$ e à "elaboração usual". O resíduo obtido foi submetido à CCS, obtendo-se $170 \mathrm{mg}(0,40 \mathrm{mmol}, 70 \%)$ de 4-Oalil-2,3-di- $O$-benzil- $\alpha$-D-galactopiranosídeo de metila (11'), que foi eluído com hexano/acetato 6:4. Óleo incolor. $[\alpha]_{\mathrm{D}}+34,6(c) 1,20$, $\left.\mathrm{CHCl}_{3}\right) . \mathbf{I V}\left(\mathrm{KBr}, \mathrm{cm}^{-1}\right) v_{\max }: 3500,3100,3050,2900,1500,1450$, 1050, 750, 700. RMN ${ }^{1} \mathbf{H}\left(200 \mathrm{MHz}, \mathrm{CDCl}_{3}\right) \delta: 7,39-7,24(\mathrm{~m}, 10 \mathrm{H}-$ aromáticos), 5,90 (dtd, $J_{8,9}=17,2 \mathrm{~Hz}, J_{8,9}=10,0 \mathrm{~Hz}, J_{8,7}=6,8 \mathrm{~Hz}$, $J_{8,7}=4,1 \mathrm{~Hz}, \mathrm{H}-8$ ), 5,22 (dd, $J_{9,8}=17,2 \mathrm{~Hz}, J_{\text {gem }}=1,5 \mathrm{~Hz}, \mathrm{H}-9$ ), 5,17 (d, $J_{9,8}=10,0 \mathrm{~Hz}, \mathrm{H}-9$ ') $, 4,83$ (d, $J_{\text {gem }}=11,8 \mathrm{~Hz}, 2$ H-benzílicos), 4,71 (d, $J_{\text {gem }}=12,0 \mathrm{~Hz}, \mathrm{H}$-benzílico), 4,70 (d, $\left.J_{1,2}=3,8 \mathrm{~Hz}, \mathrm{H}-1\right), 4,66$ (d, $J_{\text {gem }}=12,0 \mathrm{~Hz}$, H-benzílico), 4,46 (dd, $J_{\text {gem }}=12,6 \mathrm{~Hz}, J_{7,8}=4,1 \mathrm{~Hz}, \mathrm{H}-$ $7), 4,10\left(\mathrm{dd}, J_{\text {gem }}=12,6 \mathrm{~Hz}, J_{7,8}=6,8 \mathrm{~Hz}, \mathrm{H}-7\right), 3,98\left(\mathrm{dd} J_{23}=10,1 \mathrm{~Hz}\right.$, $\left.J_{2,1}=3,8 \mathrm{~Hz}, \stackrel{\mathrm{H}-2}{\mathrm{~g}}\right), 3,88\left(\mathrm{dd}, J_{3,2}=10,1 \mathrm{~Hz}, J_{34}=2,9 \mathrm{~Hz}, \mathrm{H}-3\right), 3,85-$ 3,80 (m, H-4, H-6), 3,78-3,75 (m, H-5), 3,69-3,66 (m, H-6'), 3,37 (s, $\left.\mathrm{OCH}_{3}\right), 2,23$ (s, OH). RMN ${ }^{13} \mathbf{C}\left(50 \mathrm{MHz}, \mathrm{CDCl}_{3}\right) \delta: 138,67,138,48$ (C ipso), 134,99 (C-8), 128,41, 128,36, 128,60, 127,54 (C-aromáti- $\cos ), 117,80$ (C-9), 98,86 (C-1), 78,79 (C-3), 76,49 (C-2), 75,87 (C4), 70,26 (C-5), 73,86 (C-7), 73,66, 73,40 (C-benzílicos), 62,69 (C6), 55,37 $\left(\mathrm{CH}_{3} \mathrm{O}\right)$. Análise elementar: encontrado: $\mathrm{C} 70,03 \%, H$ $7,01 \%$; requerido para $\mathrm{C}_{24} \mathrm{H}_{30} \mathrm{O}_{6}: C 69,55 \%, H 7,30 \%$.

\section{4- $\boldsymbol{O}$-alil-2,3-di- $\boldsymbol{O}$-benzil- $\alpha$-D-glicopiranosídeo de metila $(\mathbf{1 1})^{49}$}

O 4-O-alil-6-O-tert-butildimetilsilil-2,3-di-O-benzil- $\alpha$-Dglicopiranosídeo de metila (10) (300 mg, 0,57 mmol) foi submetido às mesmas condições descritas para o derivado 10', conduzindo ao 4- $O$-alil-2,3-di- $O$-benzil- $\alpha$-D-glicopiranosídeo de metila (11) (170 mg, 0,40 mmol, 70\%). Sólido branco, PF $=67,2-68,9 .[\alpha]_{D}$ $+38,4,\left(c 1,00, \mathrm{CHCl}_{3}\right)$. IV $\left(\mathrm{KBr}, \mathrm{cm}^{-1}\right) v_{\max }: 3300,2900,1470$, $1450,1200,1100,1050,750,730,700 . \mathbf{R M N}^{1} \mathbf{H}\left(200 \mathrm{MHz}, \mathrm{CDCl}_{3}\right)$ $\delta$ : 7,41-7,30 (m, $10 \mathrm{H}$-aromáticos), 5,91 (dtd, $J_{8,9}=18,0 \mathrm{~Hz}, J_{8,9}=$ $\left.10,3 \mathrm{~Hz}, J_{8,7}=J_{8,7}=5,9 \mathrm{~Hz}, \mathrm{H}-8\right), 5,24\left(\mathrm{~d}, J_{0,8}=17,4 \mathrm{~Hz}, \mathrm{H}-9\right), 5,15$ (d, $J_{9,8}=10,3 \mathrm{~Hz}, \mathrm{H}-9$ '), 4,94 (d, $J_{\text {gem }}=10,0 \mathrm{~Hz}, \mathrm{H}$-benzílico), 4,80 (d, $J_{\text {gem }}=10,0 \mathrm{~Hz}$, H-benzílico), 4,79 (d, $J_{\text {gem }}=12,0 \mathrm{~Hz}, \mathrm{H}$-benzílico), $4,64\left(\mathrm{~d}, J_{\text {gem }}=12,0 \mathrm{~Hz}, \mathrm{H}\right.$-benzílico), 4,56 (d, $\left.J_{12}=3,5 \mathrm{~Hz}, \mathrm{H}-1\right)$, $4,38\left(\mathrm{dd}, \stackrel{\mathrm{g}}{\mathrm{gem}}_{\mathrm{g}}=12,4 \mathrm{~Hz}, J_{7,8}=5,9 \mathrm{~Hz}, \mathrm{H}-7\right), 4,10\left(\mathrm{dd}, J_{\text {gem }}=12,4 \mathrm{~Hz}\right.$, $J_{7,8}=5,9 \mathrm{~Hz}, \mathrm{H}-7$ '), 3,98 (t, $\left.J_{3,2}=J_{3,4}=9,2 \mathrm{~Hz}, \mathrm{H}-3\right), 3,86$ (dd, $J_{\text {gem }}=$ $\left.11,6 \mathrm{~Hz}, J_{6,5}=2,8 \mathrm{~Hz}, \mathrm{H}-6\right), 3,76\left(\mathrm{dd}, J_{\mathrm{gem}}=11,6 \mathrm{~Hz}, J_{6,5}=3,9 \mathrm{~Hz}\right.$, H-6'), 3,74-3,63 (m, H-5), 3,50 (dd, $J_{2,3}=9,2 \mathrm{~Hz}, J_{2,1}=3,5 \mathrm{~Hz}, \mathrm{H}-$ 2), 3,48-3,36 (m, H-4), 3,42 (s, OCH ) $_{3}, 1,91(\mathrm{~s}, \mathrm{OH})$. RMN ${ }^{13} \mathbf{C}(50$ $\mathrm{MHz}, \mathrm{CDCl}_{3}$ ) $\delta: 138,98,138,37$ (C ipso), 134,96 (C-8), 128,66, 128,57, 128,29, 128,20, 128,12, 127,81 (C-aromáticos), 117,32 (C9), 98,42 (C-1), 82,01 (C-3), 80,06 (C-2), 77,90 (C-4), 70,90 (C-5), 76,63, 73,63 (C-benzílicos), 74,08 (C-7), 62,07 (C-6), 55,40 $\left(\mathrm{CH}_{3} \mathrm{O}\right)$. Análise elementar: encontrado: $C$ 69,95\%, $H$ 7,47\%; requerido para $\mathrm{C}_{24} \mathrm{H}_{30} \mathrm{O}_{6}: C 69,55 \%, H 7,30 \%$.

\section{4- $O$-alil-2,3-di- $O$-benzil-6- $O$-(2-iodobenzoil)- $\alpha$-D- galactopiranosídeo de metila $(4)^{47}$}

A uma solução de cloreto de 2-iodobenzoíla (482 mg, 1,81 mmol), preparado pela reação de ácido 2-iodobenzóico com cloreto de tionila ${ }^{50}$, em $2 \mathrm{~mL}$ de diclorometano anidro, em banho de gelo, foi adicionado $0,5 \mathrm{~mL}$ de trietilamina ( $363 \mathrm{mg}, 3,36 \mathrm{mmol})$. A mistura foi submetida à agitação magnética e, em seguida, adicionou-se, gota-a-gota, solução de 4- $O$-alil-2,3-di- $O$-benzil- $\alpha$-Dgalactopiranosídeo de metila (11') (500 mg, 1,21 mmol) em 2 mL de diclorometano anidro. Após o término da reação, a fase orgânica foi separada e a fase aquosa foi extraída com diclorometano $(3 \mathrm{x}$ $10 \mathrm{~mL}$ ). As fases orgânicas foram reunidas e lavadas com solução de $\mathrm{HCl} 3 \mathrm{~mol} / \mathrm{L}$. Em seguida, foi realizada a "elaboração usual". O resíduo obtido foi submetido à CCS e $700 \mathrm{mg}(1,09 \mathrm{mmol}, 89 \%)$ de 4-O-alil-2,3-di- $O$-benzil-6- $O$-(2-iodobenzoil)- $\alpha$-Dgalactopiranosídeo de metila (4) foram eluídos com hexano/acetato 8:2. Óleo amarelado. $[\alpha]_{\mathrm{D}}+34,4\left(c 1,00, \mathrm{CHCl}_{3}\right)$. IV $\left(\mathrm{KBr}, \mathrm{cm}^{-1}\right)$ $v_{\max }: 3100,3050,3000,2900,1750,1600,1500,1450,1250,1050$, 750, 700. RMN ${ }^{1} \mathbf{H}\left(200 \mathrm{MHz}, \mathrm{CDCl}_{3}\right) \delta: 8,00\left(\mathrm{dl}, J_{\mathrm{ed}}=8,0 \mathrm{~Hz}, \mathrm{H}-\right.$ e), 7,78 (dd, $\left.J_{\mathrm{b}, \mathrm{c}}=8,0 \mathrm{~Hz}, J_{\mathrm{b}, \mathrm{d}}=1,6 \mathrm{~Hz}, \mathrm{H}-\mathrm{b}\right), 7,41-7,26(\mathrm{~m}, 11 \mathrm{H}-$ aromáticos), 7,16 (dt, $\left.J_{\mathrm{d}, \mathrm{c}}=8,0, J_{\mathrm{d}, \mathrm{e}}=8,0 \mathrm{~Hz}, J_{\mathrm{d}, \mathrm{b}}=1,6 \mathrm{~Hz}, \mathrm{H}-\mathrm{d}\right)$, $5,95-5,87$ (m, H-8), 5,23 (dd, $J_{9,8}=17,2 \mathrm{~Hz}, J_{\text {gem }}=1,6 \mathrm{~Hz}, \mathrm{H}-9$ ), 5,15 (d, $J_{9,8}=11,2 \mathrm{~Hz}, \mathrm{H}-9$ '), 4,85 (d, $J_{\text {gem }}=12,0 \mathrm{~Hz}, \mathrm{H}-$ benzílico), $4,84\left(\mathrm{~d}, J_{\mathrm{gem}}^{9,8}=12,0 \mathrm{~Hz}, \mathrm{H}-\right.$ benzílico $), 4,72\left(\mathrm{~d}, J_{\text {gem }}=12,0 \mathrm{~Hz}, \mathrm{H}-\right.$ benzílico), 4,70 (d, $\left.J_{1,2}=3,6 \mathrm{~Hz}, \mathrm{H}-1\right), 4,67\left(\mathrm{~d}, \stackrel{J}{\mathrm{gem}}_{\mathrm{gem}}=12,0 \mathrm{~Hz}, \mathrm{H}-\right.$ benzílico), 4,50-4,40 (m, 2 H-6, H-7), 4, 13 (dd, $J_{\text {gem }}^{=}=12,4 \mathrm{~Hz}, J_{7,8}=$ $6,8 \mathrm{~Hz}, \mathrm{H}^{-7}$ '), 4,06 (t, $\left.J_{5,6}=J_{5,}=6,4 \mathrm{~Hz}, \mathrm{H}-5\right), 4,01\left(\mathrm{dd}, J_{23}=10,0\right.$ $\mathrm{Hz}, J_{2,1}=3,6 \mathrm{~Hz}, \mathrm{H}-2$ ), 3,91 (dd, $J_{3,2}=10,0 \mathrm{~Hz}, J_{3,4}=2,8 \mathrm{~Hz}, \mathrm{H}-3$ ), 3,87 (sl, H-4), 3,37 (s, $\left.\mathrm{OCH}_{3}\right)$. RMN ${ }^{13} \mathbf{C}\left(50 \mathrm{MHz}^{3,4} \mathrm{CDCl}_{3}\right) \delta: 166,26$ $(C=\mathrm{O}), 141,59$ (C-e), 138,79, 138,62 (C ipso), 135,16 (C-8), 134,85 (C-a), 132,98 (C-d), 131,13 (C-b), 128,56, 128,51, 128,20, 128,06, 
127,89, 127,76, 127,71 (C-aromáticos), 117,80 (C-9), 99,99 (C-1), 94,33 (C-f), 78,80, 76,57, 75,28, 68,33 (C-2, C-3, C-4, C-5), 74,16, 73,84, 73,58 (C-benzílicos, C-7), 64,91 (C-6), 55,65 ( $\left.\mathrm{CH}_{3} \mathrm{O}\right)$. Análise elementar: encontrado: $C 59,38 \%, H$ 6,93\%; requerido para $\mathrm{C}_{31} \mathrm{H}_{33} \mathrm{IO}_{7}: C 57,77 \%, H$ 5,16\%.

\section{4- $O$-alil-2,3-di- $O$-benzil-6- $O$-(2-iodobenzoil)- $\alpha$-D- glicopiranosídeo de metila $(3)^{47}$}

O 4-O-alil-2,3-di- $O$-benzil- $\alpha$-D-glicopiranosídeo de metila (11) (260 mg, 0,68 mmol) foi submetido às mesmas condições descritas para o derivado galacto 11' e conduziu ao 4- $O$-alil-2,3di- $O$-benzil-6- $O$-(2-iodobenzoil)- $\alpha$-D-glicopiranosídeo de metila

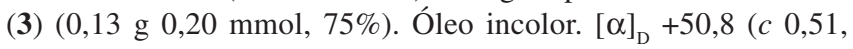
$\left.\mathrm{CHCl}_{3}\right)$. IV $\left(\mathrm{KBr}, \mathrm{cm}^{-1}\right) v_{\mathrm{max}}: 2900,1730,1600,1500,1450,1300$, 1250, 1100, 1050, 750, 700. RMN ${ }^{1} \mathbf{H}\left(200 \mathrm{MHz}_{\mathrm{m}} \mathrm{CDCl}_{3}\right) \delta: 7,99$ $\left(\mathrm{dd}, J_{\mathrm{e}, \mathrm{d}}=7,8 \mathrm{~Hz}, J_{\mathrm{e}, \mathrm{c}}=1,0 \mathrm{~Hz}, \mathrm{H}-\mathrm{e}\right), 7,80\left(\mathrm{dd}, J_{\mathrm{b}, \mathrm{c}}=7,8 \mathrm{~Hz}, J_{\mathrm{b}, \mathrm{d}}=\right.$ 1,7 Hz, H-b), 7,41-7,25 (m, 11 H-aromáticos), 7,14 (dt, $J_{\mathrm{d}, \mathrm{c}}=7,8$ $\left.\mathrm{Hz}, J_{\mathrm{d}, \mathrm{e}}=7,8 \mathrm{~Hz}, J_{\mathrm{d}, \mathrm{b}}=1,7 \mathrm{~Hz}, \mathrm{H}-\mathrm{d}\right), 5,98-5,79$ (m, H-8), 5,23 (dd, $\left.J_{9,8}=17,3 \mathrm{~Hz}, J_{\text {gem }}=1,7 \mathrm{~Hz}, \mathrm{H}-9\right), 5,13\left(\mathrm{dd}, J_{9,8}=11,2 \mathrm{~Hz}, J_{\text {gem }}=\right.$ 1,7 Hz, H-9'), 4,99-4,62 (m, 4 H-benzílicos), 4,60 (d, $J_{1,2}=3,6$ $\mathrm{Hz}, \mathrm{H}-1), 4,56\left(\mathrm{dd}, J_{\text {gem }}=11,9 \mathrm{~Hz}, J_{6,5}=2,4 \mathrm{~Hz}, \mathrm{H}-6\right), 4,48$ (dd, $J_{\text {gem }}=11,9 \mathrm{~Hz}, J_{6,5}=4,8 \mathrm{~Hz}, \mathrm{H}-6$ ') $, 4,36\left(\mathrm{dd}, J_{\text {gem }}=12,2 \mathrm{~Hz}, J_{7.8}=\right.$ $5,6 \mathrm{~Hz}, \mathrm{H}-7$ ), 4,12 (dd, $J_{\text {gem }}=12,2 \mathrm{~Hz}, J_{7,8}=6,0 \mathrm{~Hz}, \mathrm{H}-7$ '), 3,97 (t, $\left.J_{3,2}=J_{3,4}=9,2 \mathrm{~Hz}, \mathrm{H}-3\right), 3,91\left(\mathrm{ddd}, J_{5,4}=9,2 \mathrm{~Hz}, J_{5,6}=2,4 \mathrm{~Hz}, J_{5,6}=\right.$ $4,8 \mathrm{~Hz}, \mathrm{H}-5), 3,51\left(\mathrm{dd}, J_{2,3}=9,2 \mathrm{~Hz}, J_{2,1}=3,6 \mathrm{~Hz}, \mathrm{H}-2\right), 3,48-3,39$ (m, H-4), 3,39 (s, $\left.\mathrm{OCH}_{3}\right)$. RMN ${ }^{13} \mathbf{C}\left(50 \mathrm{MHz}^{2} \mathrm{CDCl}_{3}\right) \delta: 166,28$ $(C=\mathrm{O}), 141,71$ (C-e), 138,74, 138,28 (C ipso), 134,68 (C-8), 133,03 (C-a), 131,28 (C-d), 128,71, 128,67, 128,35, 128,30, 128,19, 128,13, 127,97 (C-aromáticos), 117,74 (C-9), 98,28 (C1), 94,53 (C-f), 82,12 (C-3), 80,02 (C-2), 77,83 (C-4), 68,83 (C5), 76,13, 74,24, 73,65 (C-benzílicos, C-7), 64,48 (C-6), 55,61 $\left(\mathrm{CH}_{3} \mathrm{O}\right)$. Análise elementar: encontrado: $\mathrm{C} 58,13 \%, \mathrm{H} \mathrm{4,87 \%}$; requerido para $\mathrm{C}_{31} \mathrm{H}_{33} \mathrm{IO}_{7}$ : $C 57,77 \%, H 5,16 \%$.

Reação radicalar com 4-O-alil-2,3-di- $O$-benzil-6-O-(2iodobenzoil)- $\alpha$-D-galacto-piranosídeo de metila $(4)^{28,35,36}$

A um balão bitubulado adaptado a um condensador de refluxo com tubo de nujol e um funil de adição contendo solução de 4- $O$ alil-2,3-di- $O$-benzil-6- $O$-(2-iodobenzoil)- $\alpha$-D-galactopiranosídeo de metila (4) (500 mg, 0,77 mmol) em benzeno anidro (75 mL), sob agitação magnética, refluxo e pressão positiva de nitrogênio, adicionou-se, gota-a-gota, por um período de 1,5 h, uma solução de hidreto de tributilestanho $(0,31 \mathrm{~mL}, 1,25 \mathrm{mmol})$ e AIBN $(10 \mathrm{mg})$ em benzeno anidro $(25 \mathrm{~mL})$. Terminada a adição manteve-se o refluxo por mais $1 \mathrm{~h}$. Decorrido este tempo, o solvente foi destilado e o resíduo submetido à CCS. Isolaram-se $62 \mathrm{mg}(0,12 \mathrm{mmol}, 19 \%)$ do produto de hidrogenólise (13), $30 \mathrm{mg}$ de uma mistura contendo a macrolactona $(\mathbf{4 A})$ e $86 \mathrm{mg}(0,13 \mathrm{mmol}, 17 \%)$ do material de partida 4, eluídos, respectivamente, com hexano/acetato 9:1, hexano/ acetato 8,5:1,5 e hexano/acetato 8:2.

Macrolactona (4A): $\mathbf{R M N}{ }^{1} \mathbf{H}\left(400 \mathrm{MHz}, \mathrm{CDCl}_{3}\right)$ \&: 7,76 (dl, $\left.J_{\mathrm{b}, \mathrm{c}}=7,6 \mathrm{~Hz}, \mathrm{H}-\mathrm{b}\right), 7,43-7,22$ (m, 13 H-aromáticos), 4,86 (d, $J_{\text {gem }}=$ 12,0 Hz, H-benzílico), 4,81 (d, $J_{\text {gem }}=11,7 \mathrm{~Hz}, \mathrm{H}$-benzílico), 4,74 (d, $\left.J_{1,2}=3,7 \mathrm{~Hz}, \mathrm{H}-1\right), 4,71$ (d, $J_{\text {gem }} \stackrel{\text { gem }}{=} 12,0 \mathrm{~Hz}, \mathrm{H}-$ benzílico), 4,69 (d, $J_{\text {gem }}=11,7 \mathrm{~Hz}, \mathrm{H}$-benzílico), 4,75-4,62 (m, H-6), 4,26 (dd, $J_{\text {gem }}=$ $11,7 \mathrm{~Hz}, J_{6,5}=5,7 \mathrm{~Hz}, \mathrm{H}-6$ '), 4,07-4,04 (m, H-5), 3,97-3,86 (m, H2, H-3, H-4, H-7), 3,74-3,69 (m, H-7'), 3,38 (s, CH O), 3,27-3,21 (m, H-9), 2,70-2,64 (m, H-9'), 1,98-1,93 (m, H-8), 1,82-1,77 (m, H-8'). RMN ${ }^{13} \mathbf{C}\left(100 \mathrm{MHz}, \mathrm{CDCl}_{3}\right) \delta: 169,38(C=\mathrm{O}), 142,90(\mathrm{C}-$ a), 138,61, 131,27 (C ipso), 131,80, 130,92, 129,97, 128,38, 128,34, 128,03, 127,95, 127,69, 127,59, 127,57, 126,02 (C-aromáticos),
99,05 (C-1), 79,18, 79,55, 76,39 (C-2, C-3, C-4), 66,74 (C-5), 73,70, 73,21(C-benzílicos), 70,08 (C-7), 64,32 (C-6), 55,56 ( $\left.\mathrm{CH}_{3} \mathrm{O}\right), 31,63$ (C-8), 30,52 (C-9).

Produto de hidrogenólise (13): Óleo incolor. $\mathbf{R M N}^{\mathbf{1}} \mathbf{H}(200 \mathrm{MHz}$, $\left.\mathrm{CDCl}_{3}\right) \delta: 8,04\left(\mathrm{dd}, J_{\mathrm{b}, \mathrm{c}}=7,0 \mathrm{~Hz}, J_{\mathrm{b}, \mathrm{d}}=0,8 \mathrm{~Hz}, \mathrm{H}-\mathrm{b}\right), 7,65\left(\mathrm{dd}, J_{\mathrm{d}, \mathrm{c}}=7,0\right.$ $\left.\mathrm{Hz}, J_{\mathrm{d}, \mathrm{b}}=0,8 \mathrm{~Hz}, \mathrm{H}-\mathrm{d}\right), 7,50\left(\mathrm{dt}, J_{\mathrm{c}, \mathrm{b}}=7,0 \mathrm{~Hz}, J_{\mathrm{c}, \mathrm{d}}=7,0 \mathrm{~Hz}, J_{c, c}=1,4\right.$ $\mathrm{Hz}, \mathrm{H}-\mathrm{c}), 7,38-7,28$ (m, $11 \mathrm{H}$-aromáticos), 5,91 (dddd, $J_{8,9}=17,2 \mathrm{~Hz}$, $J_{8,9}=10,3 \mathrm{~Hz}, J_{8,7}=6,7 \mathrm{~Hz}, J_{8,7}=5,4 \mathrm{~Hz}, \mathrm{H}-8$ ), 5,22 (dd, $J_{9,8}=17,2$ $\left.\mathrm{Hz}, J_{\text {gem }}=1,6 \mathrm{~Hz}, \mathrm{H}-9\right), 5,13$ (d, $J_{9,8}=10,3 \mathrm{~Hz}, J_{\text {gem }}=1,3 \mathrm{~Hz}, \mathrm{H}-9$ '), $4,86\left(\mathrm{~d}, J_{\text {gem }}=11,8 \mathrm{~Hz}, \mathrm{H}\right.$-benzílico), $4,85\left(\mathrm{~d}, J_{\text {gem }}=12,0 \mathrm{~Hz}, \mathrm{H}-\right.$ benzílico), 4,75-4,70 (m, H-benzílico, H-1), 4,62 (d, $J_{\text {gem }}=12,0 \mathrm{~Hz}$, H-benzílico), 4,50-4,40 (m, 2 H-6, H-7), 4,14 (dd, $J_{\text {gem }}^{\text {em }}=11,8 \mathrm{~Hz}$, $J_{7}, 8=6,7 \mathrm{~Hz}, \mathrm{H}-7$ ') , 4,05-3,87 (m, H-2, H-3, H-4, H-5), 3,37 (s, $\mathrm{CH}_{3} \mathrm{O}$ ). RMN ${ }^{13} \mathbf{C}\left(50 \mathrm{MHz}, \mathrm{CDCl}_{3}\right) \delta: 168,35(\mathrm{C}=\mathrm{O}), 147,99(\mathrm{C}-\mathrm{a}), 138,89$, 138,69 (C-ipso), 137,32 (C-8), 135,27, 132,11, 129,75, 128,59, 128,28, 128,10, 127,95, 127,75 (C-aromáticos), 117,74 (C-9), 98,99 (C-1), 79,04, 76,67, 75,30, 68,48 (C-2, C-3, C-4, C-5), 74,24, 73,92, 73,66 (C-benzílicos, C7), 64,10, (C-6), 55,51 ( $\left.\mathrm{CH}_{3} \mathrm{O}\right)$.

Reação radicalar com 4-O-alil-2,3-di- $O$-benzil-6- $O$-(2iodobenzoil)- $\alpha$-D-glicopiranosídeo de metila $(3)^{28,35,36}$

Submetendo-se o derivado $3(0,40 \mathrm{~g}, 0,62 \mathrm{mmol})$ às mesmas condições descritas para o derivado galacto 4, obteve-se o 4-Oalil-2,3-di-O-benzil-6-O-benzoil- $\alpha$-D-glicopiranosídeo de metila (12) (95 mg, 0,18 mmol, 30\%). Óleo incolor. RMN ${ }^{1} \mathbf{H}(200 \mathrm{MHz}$, $\left.\mathrm{CDCl}_{3}\right) \delta: 8,02\left(\mathrm{dd}, J_{\mathrm{b}, \mathrm{c}}=8,0 \mathrm{~Hz}, J_{\mathrm{b}, \mathrm{d}}=1,6 \mathrm{~Hz}\right.$ e H-b'), 7,59-7,19 (m, $13 \mathrm{H}$-aromáticos), 5,87 (dddd, $J_{8,9}=17,2 \mathrm{~Hz}, J_{8,9}=10,3 \mathrm{~Hz}, J_{8,7}=$ $\left.5,5 \mathrm{~Hz}, J_{8,7}=5,8 \mathrm{~Hz}, \mathrm{H}-8\right), 5,21$ (dd, $J_{9,8}=17,2 \mathrm{~Hz}, J_{\text {gem }}=1,6 \mathrm{~Hz}, \mathrm{H}-$ 9), 5,11 (dd, $J_{9,8}=10,3 \mathrm{~Hz}, J_{\text {gem }}=1,6 \mathrm{~Hz}, \mathrm{H}-9$ '), 4,99-4,63 (m, $4 \mathrm{H}-$ benzílicos), 4,61 (d, $\left.J_{1,2}=3,6 \mathrm{~Hz}, \mathrm{H}-1\right), 4,58\left(\mathrm{dd}, J_{\text {gem }}=12,0 \mathrm{~Hz}\right.$, $J_{6,5}=2,3 \mathrm{~Hz}, \mathrm{H}-6$ ), 4,47 (dd, $J_{\text {gem }}=12,0 \mathrm{~Hz}, J_{6,5}=4,6 \mathrm{~Hz}, \mathrm{H}-6$ '), $4,36\left(\mathrm{dd}, J_{\mathrm{gem}}=12,3 \mathrm{~Hz}, J_{7,8}=5,6 \mathrm{~Hz}, \mathrm{H}-7\right), 4,11\left(\mathrm{dd}, J_{\mathrm{gem}}=12,3 \mathrm{~Hz}\right.$, $\left.J_{7,8}=5,8 \mathrm{~Hz}, \mathrm{H}-7\right), 3,97\left(\mathrm{t}, J_{3,2}=J_{3,4}=9,2 \mathrm{~Hz}, \mathrm{H}-3\right), 3,91(\mathrm{~m}, \mathrm{H}-5)$, 3,53 (dd, $\left.J_{2,3}=9,2 \mathrm{~Hz}, J_{2,1}=3,6 \mathrm{~Hz}, \mathrm{H}-2\right), 3,46$ (m, H-4), 3,32 (s, $\left.\mathrm{CH}_{3} \mathrm{O}\right) . \mathbf{R M N}{ }^{13} \mathbf{C}\left(50 \mathrm{MHz}, \mathrm{CDCl}_{3}\right) \delta: 166,49(\mathrm{C}=\mathrm{O}), 138,77$, 138,32 (C-ipso), 134,68 (C-8), 133,29, 130,16, 129,84, 128,69, 128,65, 128,61, 128,36, 128,26, 128,15, 127,95 (C-aromáticos), 117,62 (C-9), 98, 27 (C-1), 82,18, 80,15, 77,87, 68,98 (C-2, C-3, C4, C-5), 76,16, 74,21, 73,65 (C-benzílicos, C7), 63,77, (C-6), 55,44 $\left(\mathrm{CH}_{3} \mathrm{O}\right)$.

\section{CONCLUSÕES}

Tentativas de síntese de macrolactonas por meio de reações radicalares foram realizadas com os orto-aliloxibenzoésteres 3 e 4 . No entanto, ao contrário do que ocorreu com os análogos benzamidas, não foram isolados os macrociclos desejados. Possivelmente estes diferentes resultados se devem ao fato de que nos benzoésteres a rotação da ligação C-O é livre, havendo radicais em várias conformações. Além disso, com relação aos confôrmeros relacionados à ligação C6-C5, não se observa nenhum tipo de estabilização da conformação adequada para ciclização. Devido à restrição de rotação da ligação $\mathrm{C}-\mathrm{N}$ das benzamidas, os radicais arila são formados predominantemente em uma das conformações. Além disso, em relação aos rotâmeros relativos à ligação C6-C5, a conformação apropriada para o ataque do radical arila à ligação dupla da cadeia lateral deve ser estabilizada por ligação de hidrogênio.

\section{AGRADECIMENTOS}

Ao CNPq pelo suporte financeiro. M. A. F. Prado e R. J. Alves agradecem ao $\mathrm{CNPq}$ pelas bolsas de produtividade em pesquisa e 
D. F. Dias agradece à FAPEMIG pela concessão da bolsa de iniciação científica e à CAPES pela concessão da bolsa de mestrado.

\section{REFERÊNCIAS}

1. Bowman, W. R.; Mann, M.; Parr, J.; J. Chem. Soc., Perkin Trans. 12000 291.

2. Escolano, C; Jones, K.; Tetrahedron Lett. 2000, 41, 8951.

3. Porter, N. A.; Chang, V. H. T.; J. Am. Chem. Soc. 1987, 109, 4976.

4. Perkins, M. J.; Radical Chemistry, New York: Ellis Horwood, 1995.

5. Curran, D. P.; Synthesis 1988, 417.

6. Jessop, C. N.; Parsons, A. F.; Routledge, A.; Irvine, D.; Tetrahedron Lett. 2003, 44, 479.

7. Allin, S. M.; Barton, W. R. S.; Bowman, W. R.; McInally, T.; Tetrahedron Lett. 2002, 43, 4191.

8. Ishibashi, H.; Sato, T.; Ikeda, M.; Synthesis 2002, 695.

9. Ishibashi, H.; Kawanami, H.; Ikeda; M.; J. Chem. Soc., Perkin Trans. 1 1997, 817.

10. Jones, K.; Wilkinson, J.; J. Chem. Soc., Chem. Commun. 1992, 1767.

11. Schultz, A. G.; Holoboski, M. A.; Smith, M. S.; J. Am. Chem. Soc. 1993, 115, 7904.

12. Jones, K.; Wilkinson, J.; Ewin, R.; Tetrahedron Lett. 1994, 35, 7673.

13. Beckwith, . A. L. J.; Gara, W.; J. Chem. Soc, Perkin Trans. 2 1975, 593.

14. Brunton, S. A.; Jones, K.; J. Chem. Soc., Perkin Trans. 1 2000, 763.

15. Ishibashi, H.; Kato, I. ; Takeda, Y.; Kogure, M.; Tamura, O.; Chem. Commun. 2000, 1527.

16. Ishibashi, H.; Kobayashi, T.; Machida, N.; Tamura, O.; Tetrahedron 2000, $56,1469$.

17. Ishibashi, H.; Kato, I. ; Takeda, Y.; Tamura, O.; Tetrahedron Lett. 2001, $42,931$.

18. Borger, D. L.; Boyce, C. W.; J. Org. Chem. 2000, 65, 4088.

19. Jia, G.; Lown, J. W.; Bioorg. Med. Chem. 2000, 8, 1607.

20. Gibson, S. E.; Guillo, N.; Tozer, M. J.; J. Chem. Soc., Chem. Commun. 1997, 637.

21. Ghosh, A. K.; Ghosh, K.; Pal, S.; Ghatak, U. R.; J. Chem. Soc., Chem Commun. 1993, 809

22. Ghosh, K.; Ghosh, A. K ; Ghatak, U. R.; J. Chem. Soc., Chem. Commun. 1994, 629 .

23. Ghosh, K.; Ghatak, U. R.; Tetrahedron Lett. 1995, 36, 4897.
24. Chattopadhayay, P.; Mukherjee, M.; Ghosh, S.; Chem. Commun. 1997, 2139.

25. Nandi, A.; Mukhopadhyay, R.; Chattopadhyay. P.; J. Chem. Soc., Perkin Trans. 1 2001, 3346.

26. Nandi, A.; Chattopadhyay. P.; Tetrahedron Lett. 2002, 43, 5977.

27. Lamas, C.; Saá, L.; Castedo, L.; Domínguez, D.; Tetrahedron Lett. 1992, 33,5653 .

28. Baldwin, J. E.; Adlington, R. M.; Ramcharitar, S. H.; Tetrahedron 1992, $48,3413$.

29. Snieckus, V.; Cuevas, J. C.; Sloan, C. P.; J. Am. Chem. Soc. 1990, 112, 896.

30. Abeywickrema, A. N.; Beckwith A. L. J.; J. Chem. Soc., Chem. Commun. 1986, 464.

31. Beckwith A. L. J.; Glover, S. A.; Aust. J. Chem. 1987, 40, 157.

32. Beckwith A. L. J.; Bowry, V. W.; Moad, G.; J. Org. Chem. 1988, 53, 1632.

33. Prado, M. A. F.; Alves, R. J.; Souza Filho, J. D.; Alves, R. B.; Pedrosa, M. T. C.; Prado, R. F.; Faraco, A. A. G.; J. Chem. Soc., Perkin Trans. 1. 2000, 1853.

34. Binatti, I.; Prado, M. A. F.; Alves, R. J.; Souza-Filho, J. D.; J. Braz. Chem. Soc. 2002, 13, 570

35. Beckwith, A. L. J.; Drok, K.; Mailard, B. Degueil-castaing, M.; Philippon, A.; J. Chem. Soc., Chem. Commun. 1997, 499.

36. Marinovic, N. N.; Ramanathan, H.; Tetrahedron Lett. 1983, 24, 1871.

37. Curran, D. P.; Chang, C.; J. Org. Chem. 1989, 54, 3140.

38. Curran, D. P.; Tamine, J.; J. Org. Chem. 1991,56, 2746.

39. Lewin, A. H.; Frucht, M.; Chen, K. V. J.; Tetrahedron 1975, 31, 207.

40. Faraco, A. A. G.; Prado, M. A. F.; Alves, R. J.; Souza-Filho, J. D.; Alves, R. B.; Faraco, R. F. P.; Synth. Commun. 2003, 33, 463.

41. Faraco, A. A. G.; Tese de Doutorado, Universidade Federal de Minas Gerais, Brasil, 2001.

42. Hall, D. M.; Carbohyd. Res. 1980, 86, 158

43. Robertson, J. G.; Lamb, A. R.; J. Chem. Soc. 1934, 1321

44. Fréchet, M. J.; Baer, H. H., Can. J. Chem. 1975, 54, 67.

45. Bell, D. J.; Lorber, J. ; J. Chem. Soc. 1940, 453.

46. Kiss, J.; Burkhardt, S.; Helv. Chim. Acta 1970, 53, 1000

47. Dufour, M.; Gramain, J. C.; Husson, H. P.; Sinibaldi, M. E.; Troyn, Y.; Synth. Commun. 1992, 22, 189.

48. Pietraszkiewicz, M.; Jurczak, J.; Tetrahedron 1984, 40, 2967.

49. Corey, E. J.; Venkateswarlu, A.; J. Am. Chem. Soc. 1972, 94, 6190.

50. Beak, P.; Musick, T. J.; Chen, C.; J. Am. Chem. Soc. 1988, 110, 3538 